\title{
General Relativistic Shock Waves in Fluids for which Pressure Equals Energy Density
}

\author{
A. H. Taub \\ Mathematics Department, University of California, Berkeley, California, USA
}

Received August 10, 1972

\begin{abstract}
A shock wave in a self-gravitating fluid obeying the equation of state: pressure equal to energy density is shown to travel with the velocity of light in a space-time determined by the Einstein field equations. The jump conditions that must be satisfied by the hydrodynamic variables are derived and discussed as are those that must be satisfied by the metric tensor and its derivatives. The latter conditions are obtained by using a variational principle.
\end{abstract}

\section{Introduction}

The preceding paper [1] discussed some solutions of the Einstein field equations for the case where the source of the gravitational field was a perfect fluid described by the stress energy tensor

$$
T^{\mu v}=(w+p) u^{\mu} u^{v}-p q^{\mu v}
$$

with $u^{\mu}$ the normalized four-velocity vector, that is, with

$$
u^{\mu} u_{\mu}=1,
$$

$w$ the rest energy density and $p$ the pressure. It was also assumed that the fluid obeyed the "extreme" equation of state

$$
w=p .
$$

It is the purpose of this paper to discuss the jump conditions that must be satisfied across a singular hypersurface, such as a shock wave, in the space-time determined by the Einstein field equations when the source of the gravitational field is the extreme fluid described by Eqs. (1.1) through (1.3). These jump conditions are of two sorts: (1) conditions that must be satisfied by the hydrodynamic variables, to so-called Rankine-Hugoniot equations and (2) conditions that must be satisfied by the metric tensor and its derivatives. 
The former conditions may be obtained from a reformulation of the conservation equations satisfied by the hydrodynamic variables. The next section will start the discussion of the Rankine-Hugoniot equations. The treatment of the conditions on the metric tensor will begin in Section 4 and will make use of the variational method described in [2]. This method may be summarized as follows: Suppose the Einstein field equations in a region of space-time removed from the singular hypersurface may be derived from the variational principle characterized by the integral

$$
I=\int_{V}(R-2 k \mathscr{L}) \sqrt{-g} d^{4} x
$$

where $R$ is the scalar curvature of space-time, $\mathscr{L}$ is the Lagrangian characterizing the source of the gravitational field and $V$ is an arbitrary four-volume in space-time containing the singular hypersurface. We then assume that when the singular hypersurface $\Sigma$ divides the four volume $V$ into two subregions $V_{1}$ and $V_{2}$ the integral may be written as

where

$$
I=I_{1}+I_{2}
$$

$$
I_{A}=\int_{V_{A}}(R-2 k \mathscr{L}) \sqrt{-g} d^{4} x \quad(A=1,2) .
$$

It is required that $I$ be an extremal for arbitrary variations of the metric tensor, the source variables and constrained variations of the hypersurface $\Sigma$. We shall only treat the case of shock waves; that is, we shall assume that when $\mathscr{L}$ is integrated over the volume $V$ it does not produce a term involving an integral over the hypersurface $\Sigma$. The latter would be the situation if an infinitely thin shell were present.

\section{Thermodynamic Properties of the Extreme Fluid}

These properties are often described in terms of the pressure $p$ and the rest particle density of the fluid, $\varrho$. The latter quantity is required to satisfy the conservation of particle number equation,

$$
\left(\varrho u^{\mu}\right)_{; \mu}=0
$$

and serves to define the internal energy $\varepsilon$ through the equation

$$
w=\varrho(1+\varepsilon)
$$

where we have chosen our units so that the special relativity velocity of light

$$
c=1 \text {. }
$$

Eqs. (1.3) and (1.2) then imply that

$$
\varepsilon=p / \varrho-1 .
$$


The conservation equations

$$
T^{\mu v} ; v=0
$$

together with Eqs. (1.0) and (2.2) then imply that

$$
\varrho T u^{\mu},_{\mu}=0
$$

where the temperature $T$ and the entropy $S$ are defined by the equation

$$
T d S=d \varepsilon+p d\left(\frac{1}{\varrho}\right) .
$$

In case Eq. (2.3) holds we find that

and

$$
T=p / \varrho
$$

$$
k S=\log \frac{p}{\varrho^{2}} .
$$

That is, the extreme fluid behaves as a fluid with a ratio of specific heats

$$
\gamma=2 \text {. }
$$

\section{The Rankine-Hugoniot Equations}

These equations are derived from the generalization of Eqs. (2.1) and (2.4) which are

and

$$
\begin{aligned}
& {\left[\varrho u^{\mu} n_{\mu}\right]=0} \\
& {\left[T^{\mu v} n_{\mu}\right]=0}
\end{aligned}
$$

where $n_{\mu}$ is a vector normal to the hypersurface and we have used the notation

$$
[f]=\lim _{\varepsilon \rightarrow 0}\left(f\left(x^{\mu}+\varepsilon n^{\mu}\right)-f\left(x^{\mu}-\varepsilon n^{\mu}\right)\right)=f_{+}-f_{-} .
$$

Thus Eqs. (3.1) and (3.2) may be written as

and

$$
\varrho_{+} u_{+}^{\mu} n_{\mu}=\varrho_{-} u_{-}^{\mu} n_{\mu}=M
$$

when

$$
\begin{aligned}
p_{+}\left(2 u_{+}^{\mu} u_{+}^{v} n_{v}-n^{\mu}\right) & =p_{-}\left(2 u_{-}^{\mu} u_{-}^{v} n_{v}-n^{\mu}\right) \\
g_{+}^{\mu \nu} & =g_{-}^{\mu \nu} .
\end{aligned}
$$

Our subsequent discussion will show that a coordinate system exists in which the latter equations hold.

When Eqs. (3.4) are multiplied by $n_{\mu}$ and summed we obtain

$$
2 M^{2}\left(\frac{p_{+}}{\varrho_{+}^{2}}-\frac{p_{-}}{\varrho_{-}^{2}}\right)=\left(p_{+}-p_{-}\right) n_{\mu} n^{\mu} .
$$


If Eqs. (3.4) are multiplied successively by $u_{+}^{\mu}$ and $u_{-}^{\mu}$ and $u_{+}^{\mu} u_{-} \mu$ is eliminated from the resulting equations we obtain

$$
M^{2}\left(\frac{p_{-}}{\varrho_{-}^{2}}-\frac{p_{+}}{\varrho_{+}^{2}}\right)=0=\left(p_{+}-p_{-}\right) n_{\mu} n^{\mu} .
$$

A singular hypersurface for which

$$
M=0
$$

is said to be a slip hypersurface if $\varrho_{+}$and $\varrho_{-}$do not vanish. In this case $u_{+}^{\mu}$ and $u_{-}^{\mu}$ are orthogonal to the normal to the hypersurface. The situation $M=0$ also arises when the singular hypersurface bounds a region where $\varrho_{+}=0$. In this case it follows from the second of Eqs. (3.6) that

where $n^{\mu} n_{\mu} \neq 0$.

$$
p_{+}=p_{-},
$$

A singular hypersurface for which

and

$$
M \neq 0
$$

$$
p_{+} \neq p_{-}
$$

is said to be a shock wave. In this case we have

and

$$
\frac{p_{-}}{\varrho_{-}^{2}}=\frac{p_{+}}{\varrho_{+}^{2}}
$$

$$
n_{\mu} n^{\mu}=0 \text {. }
$$

That is for a shock wave the singular hypersurface is a null hypersurface and across this hypersurface the entropy is conserved. Thus shock waves in the material described by the caloric equation of state given by Eq. (2.3) behave quite differently from those obeying more realistic caloric equations of state. For the latter materials the specific entropy of the material increases it passes through a shock wave.

In case Eqs. (3.7) and (3.8) obtain it follows that

$$
u_{-}^{\mu} n_{\mu}=\frac{\varrho_{+}}{\varrho_{-}} u_{+}^{\mu} n_{\mu}=\sqrt{\frac{p_{+}}{p_{-}}} u_{+}^{\mu} n_{\mu} .
$$

Thus the change in particle density and the change in velocity normal to the hypersurface are determined, once the ratio in pressures $p_{+} / p_{-}$ is known. It is a consequence of Eqs. (3.4) that if $Y_{\mu}$ is a vector orthogonal to $n_{\mu}$, that is if

when $M \neq 0$.

$$
\begin{aligned}
Y^{\mu} n_{\mu} & =0 \\
\left(Y_{\mu} u_{-}^{\mu}\right) & =\frac{\varrho_{+}}{\varrho_{-}}\left(Y_{\mu} u_{+}^{\mu}\right)=\sqrt{\frac{p_{+}}{p_{-}}}\left(Y_{\mu} u_{+}^{\mu}\right)
\end{aligned}
$$


In the above discussion we have assumed that the equation of state $w=p$ holds on both sides of the singular hypersurface and have shown that if this hypersurface is a shock wave then it is a null hypersurface. If we assume that the singular hypersurface is a null hypersurface it follows from Eqs. (3.2) that

where

$$
\left(w_{+}+p_{+}\right) \alpha_{+}^{2}=\left(w_{-}+p_{-}\right) \alpha_{-}^{2},
$$

when

and that

$$
\begin{gathered}
\alpha_{ \pm}=u_{ \pm}^{\mu} n_{\mu} . \\
u_{+}^{\mu} u_{-}^{v}\left(n_{\mu} Y_{v}-n_{v} Y_{\mu}\right)=0,
\end{gathered}
$$

$$
Y_{\mu} n^{\mu}=0
$$

The last equation is the Hugoniot curve which relates the thermodynamic state of the material on both sides of a shock wave travelling with the velocity of light.

If Eqs. (3.11) hold in addition to Eqs. (3.2) with $n_{\mu}$ a null vector then Eq. (3.9) may be written as

where

$$
\begin{gathered}
\tau_{+}=\tau_{-} \\
\tau=\frac{w+p}{\varrho^{2}}
\end{gathered}
$$

and is the thermodynamic variable used by Lichnerowicz [3] in his discussion of compressibility assumptions.

It is an immediate consequence of Eqs. (3.2) that a singular null hypersurface cannot separate a region of space time (say the + region) where the stress energy tensor is non-vanishing and is given by Eqs. (1.1), and (1.2) from a vacuum region one (the - region). For in such a case Eqs. (3.2) become

$$
\left(w_{+}+p_{+}\right) u_{+}^{\mu} \alpha_{+}-p_{+} n^{\mu}=0 .
$$

It follows from this equation and the assumptions made above that

and

$$
\left(w_{+}+p_{+}\right) \alpha_{+}^{2}=0
$$

$$
p_{+}=0 \text {. }
$$

Hence since $u_{+}^{\mu}$ is a time-like vector, $\alpha_{+} \neq 0$ and we must have $w_{+}=p_{+}=0$. If the assumption that $u_{+}^{\mu}$ is a time-like vector is relaxed, we see that Eqs. (3.2) imply that it must be proportional to the nullvector $n^{\mu}$.

\section{The Special Coordinate System}

We now turn to a discussion of the conditions that must be satisfied by the metric tensor and its derivatives across a singular null hypersurface $\Sigma$. The derivation of these conditions will be carried out in a 
coordinate system adapted to $\Sigma$. This coordinate system is such that the hypersurface $\Sigma$ is given by the equation

and on $\Sigma$

$$
x^{4}=0
$$

$$
\begin{aligned}
g^{\mu \nu}\left(x^{1}, x^{2}, x^{3}, 0\right)=g_{0}^{\mu \nu} & =\delta_{3}^{\mu} \delta_{4}^{v}+\delta_{4}^{\mu} \delta_{3}^{v}+\gamma^{A B} \delta_{A}^{\mu} \delta_{B}^{v} \\
g_{0 \mu \nu} & =\delta_{\mu}^{3} \delta_{v}^{4}+\delta_{v}^{3} \delta_{\mu}^{4}+\gamma_{A B} \delta_{\mu}^{A} \delta_{v}^{B} \quad(A, B=1,2)
\end{aligned}
$$

and

where

$$
\left(g^{44},{ }_{4}\right)_{0}=\left(g^{3 v},{ }_{4}\right)_{0}=0
$$

$$
\gamma_{A B} \gamma^{B C}=\delta_{A}^{C}
$$

and the subscript zero denotes the fact that a quantity is evaluated on the hypersurface $\Sigma$, that is for $x^{4}=0$.

Such a coordinate system can be constructed from an arbitrary one with coordinates $x^{\mu}$ in which the hypersurface $\Sigma$ is a member of a family of null hypersurfaces given by the equations

$$
F\left(\bar{x}^{\mu}\right)=\text { constant }
$$

as follows. The null-vector field

satisfies

$$
\bar{k}_{\mu}=F,_{\mu}
$$

The equations

$$
\bar{g}^{\mu \nu} \bar{k}_{\mu} \bar{k}_{v}=0 .
$$

$$
\bar{k}^{\mu} \phi,{ }_{\mu}=\bar{g}^{\mu v} F,{ }_{\nu} \phi,{ }_{\mu}=0
$$

admit as solutions two functions $\phi^{A}(A=1,2)$ which are independent of each other and of the function $F$. Let $f\left(\bar{x}^{\mu}\right)$ be a solution of the equations

$$
\bar{k}^{\mu} f,_{\mu}=1 \text {. }
$$

Then the transformation

is such that

$$
\begin{aligned}
& x^{\prime 4}=F(\bar{x}) \\
& x^{\prime 3}=f(\bar{x}) \\
& x^{\prime A}=\phi^{A}(\bar{x})
\end{aligned}
$$

$$
g^{\prime 4 v}=\delta_{3}^{v}=k^{\prime v} .
$$

If this transformation is followed by the transformation

$$
\begin{aligned}
x^{\prime \prime 4} & =x^{\prime 4} \\
x^{\prime \prime} & =x^{\prime i}+x^{4} f^{i}\left(x^{j}\right) \quad i, j=1,2,3
\end{aligned}
$$

we may choose the $f^{i}\left(x^{j}\right)$ so that

$$
g_{0}^{\prime \mu \nu}=\delta_{3}^{\mu} \delta_{4}^{v}+\delta_{3}^{v} \delta_{4}^{\mu}+g_{0}^{\prime \prime A B} \delta_{A}^{\mu} \delta_{B}^{v} .
$$


Finally we make the transformation

$$
x^{\mu}=x^{\mu \mu}+\frac{\left(x^{\prime \prime}\right)^{2}}{2} \phi^{\mu}\left(x^{i}\right)
$$

and choose the $\phi^{\mu}$ so that Eqs. (4.2) and (4.3) are satisfied.

In the coordinate system in which Eqs. (4.1)-(4.3) hold the vector field

$$
k_{\mu}=\delta_{\mu}^{4}
$$

satisfies the equations

Hence

$$
\left(k^{\mu ; v}\right)_{0}=\frac{1}{2}\left(g_{0,4}^{4 \mu} \delta_{3}^{v}+g_{0,4}^{4 v} \delta_{3}^{\mu}-g_{0,3}^{\mu \nu}\right)=\left(k^{v ; \mu}\right)_{0} .
$$

$$
\begin{aligned}
& \left(k^{4 ; v}\right)_{0}=0 \\
& \left(k^{3 ; 3}\right)_{0}=0 \\
& \left(k^{3 ; A}\right)_{0}=\frac{1}{2} g_{0,4}^{4 A} \\
& \left(k^{A ; B}\right)_{0}=-\frac{1}{2} \gamma_{, 3}^{A B} .
\end{aligned}
$$

In this coordinate system the vector field whose components are given by

satisfies

$$
m_{\mu}=\delta_{\mu}^{3}
$$

and

$$
m_{0}^{\mu}=\delta_{4}^{\mu}
$$

$$
\begin{aligned}
k_{0}^{\mu} m_{0 \mu} & =1 \\
m_{0}^{\mu} m_{0 \mu} & =0 .
\end{aligned}
$$

The tensor

$$
h_{0}^{\mu v}=\gamma^{A B} \delta_{A} \delta_{B}^{v}
$$

projects any vector into the two dimensional space orthogonal to the two-plane spanned by the vectors $m_{0 \mu}$ and $k_{0 \mu}$. We may write

$$
h_{0 v}^{\mu}=\delta_{C}^{\mu} \delta_{v}^{C}=\delta_{1}^{\mu} \delta_{v}^{1}+\delta_{2}^{\mu} \delta_{v}^{2} .
$$

Hence it follows from Eqs. (4.4) that

$$
A^{\mu \nu}=\left(k^{\sigma ; \tau}\right)_{0} h_{0 \sigma}^{\mu} h_{0 \tau}^{v}=-\frac{1}{2} \gamma_{, 3}^{A B} \delta_{A}^{\mu} \delta_{B}^{v} .
$$

The rate of expansion, rotation tensor, and shear tensor of the vector field $k_{\mu}$ on the hypersurface $\Sigma$ are then defined as

$$
\begin{aligned}
\theta= & A_{\mu}^{\mu} \\
= & \omega^{\mu v}=\frac{1}{2}\left(A^{\mu v}-A^{v \mu}\right) \\
& \sigma^{\mu v}=\frac{1}{2}\left(A^{\mu v}+A^{v \mu}-h^{\mu v} A_{\mu}^{\mu}\right) .
\end{aligned}
$$


Thus

$$
\begin{aligned}
\theta & =-\frac{1}{2} \gamma_{, 3}^{A B} \gamma_{A B}=\frac{1}{\sqrt{\gamma}}(\sqrt{\gamma})_{, 3}=\left(k^{\sigma ; \tau}\right)_{0} g_{\sigma \tau} \\
\omega_{\mu \nu} & =0 \\
\sigma^{\mu \nu} & =-\frac{1}{2 \sqrt{\gamma}}\left(\sqrt{\gamma} \gamma^{A B}\right)_{, 3} \delta_{A}^{\mu} \delta_{B}^{v} \\
\gamma & =\operatorname{det}\left\|\gamma_{A B}\right\|=-\operatorname{det}\left\|g_{0 \mu \nu}\right\|=-g_{0} .
\end{aligned}
$$

\section{The Jump Conditions for the Metric Tensor}

These conditions are obtained by studying the contributions to the variation of $I$ due to the divergence terms in the variation of the scalar curvature produced when the metric tensor has the variation $\dot{g}_{\mu v}$. These terms are given by

$$
J=\int_{V_{1}+V_{2}} \sqrt{-g}\left(g^{\mu v} g^{\varrho \sigma}-g^{\varrho \mu} g^{v \sigma}\right) \dot{g}_{\mu v ; \varrho \sigma} d^{4} x
$$

where we use the notation

$$
\int_{V_{1}+V_{2}} f d^{4} x=\int_{V_{1}} f d^{4} x+\int_{V_{2}} f d^{4} x .
$$

we may write

$$
J=\int_{\Sigma}\left[\sqrt{-g}\left(g^{\mu v} g^{\varrho \sigma}-g^{\varrho \mu} g^{v \sigma}\right) \dot{g}_{\mu v ; \varrho} k_{\sigma}\right] d^{3} x
$$

where

$$
k_{\sigma} d^{3} x=\delta_{\sigma}^{4} d x^{1} d x^{2} d x^{3}
$$

and the coordinate system is such that Eqs. (4.1) through (4.3) hold.

Eq. (5.1) may also be written as

$$
\begin{aligned}
J= & \int_{\Sigma}\left[\left\{\sqrt{-g}\left(g^{\mu v} g^{\varrho \sigma}-g^{\varrho \mu} g^{v \sigma}\right) k_{\sigma} \dot{g}_{\mu v}\right\}_{, \varrho}\right] d^{3} x \\
& -\int_{\Sigma}\left[\sqrt{-g}\left(g^{\mu v} g^{\varrho \sigma}-g^{\varrho \mu} g^{v \sigma}\right) \dot{g}_{\mu v k_{\sigma ;}}\right] d^{3} x
\end{aligned}
$$

where the comma again represents the partial derivative with respect to the variable indicated. We may assume that the variations $\dot{g}_{\mu \nu}$ vanish outside a compact region in the hypersurface $\Sigma$ bounded by a two surface $S$. In view of Eqs. (5.2), (4.2) and (2.6) we may write Eq. (5.3) as

$$
-J=\int[\sqrt{\gamma}] \dot{g}_{33,4} d^{3} x+\int\left[\left(\sqrt{\gamma} g^{4 \mu} g^{4 v}\right)_{, 4}+\sqrt{\gamma}\left(\theta g^{\mu \nu}-k^{\mu ; \nu}\right)\right] \dot{g}_{\mu \nu} d^{3} x
$$


The hypersurface integral $J$ will vanish for arbitrary $\dot{g}_{\mu \nu}$ and $\dot{g}_{\mu v, 4}$ which vanish on $S$ if and only if

$$
\begin{aligned}
{[\sqrt{\gamma}] } & =0, \\
{[\sqrt{\gamma} \theta] } & =0, \\
{\left[(\sqrt{\gamma})_{, 4}\right] } & =0, \\
{\left[g_{, 4}^{4 A} \sqrt{\gamma}\right] } & =0, \\
{\left[\sqrt{\gamma}\left(\theta \gamma^{A B}+\frac{1}{2} \gamma_{, 3}^{A B}\right)\right] } & =0 .
\end{aligned}
$$

These equations are obtained by setting the coefficients of $\dot{g}_{33,4}, \dot{g}_{44}$, $\dot{g}_{33}, \dot{g}_{3 A}$ and $\dot{g}_{A B}$ respectively equal to zero. The coefficients of the remaining components if $\dot{g}_{\mu v}$ vanish in the coordinate system used.

Eq. (5.8) may be written as

Since

$$
\frac{1}{2}\left[\sqrt{\gamma}\left(\gamma^{C D} \gamma^{A B}-\gamma^{C A} \gamma^{D B}\right) \gamma_{C D, 3}\right]=0 .
$$

$$
\gamma\left(\gamma^{A B} \gamma^{C D}-\gamma^{A C} \gamma^{B D}\right)=\varepsilon^{A D} \varepsilon^{B C}
$$

where $\varepsilon^{12}=\varepsilon^{21}=1$ and $\varepsilon^{11}=\varepsilon^{22}=0$, and since Eq. (5.4) holds, we must have

$$
\left[\gamma_{A B, 3}\right]=0 \text {. }
$$

Also in view of Eq. (5.4) we must have

$$
[\theta]=0 \text {. }
$$

That is the shear and the rate of expansion of the vector $k_{\mu}$ must be continuous across $\Sigma$. Eq. (5.4) implies that the $\sqrt{\gamma}$ is continuous across $\Sigma$.

We may further specialize the coordinate system so that

$$
\left[\gamma_{A B}\right]=0
$$

for on the two surface in $\Sigma$ determined by the equation $x^{3}=x^{3}$ $x^{3}=x_{0}^{3}=$ constant, $\gamma_{+A B}$ and $\gamma_{-A B}$ are the induced metrics. We may introduce coordinates such that

$$
\gamma_{ \pm A B}\left(x^{1}, x^{2} x_{0}^{3}\right)=\Phi_{ \pm}^{2}\left(x^{1}, x^{2} x_{0}^{3}\right) \delta_{A B} .
$$

The requirement that

then implies that

$$
\gamma_{+}=\gamma_{-}
$$

Hence

$$
\Phi_{+}^{2}=\Phi_{-}^{2} .
$$

$$
\left[\gamma_{A B}\right]=0
$$

on this two surface and since this quantity is independent of $x^{3}$ it vanishes everywhere on $\Sigma$. Thus we may introduce a coordinate system such that $g_{\mu \nu}$ is continuous across $\Sigma$ and satisfies Eqs. (4.2) and (4.3). 
In such a coordinate system Eqs. (5.6) and (5.7) are equivalent to the equation

$$
k_{0 \mu}\left[\left(\sqrt{-g} g^{\mu \nu}\right)_{, 4}\right]=0 .
$$

This is in turn equivalent to the equation

where

$$
k_{0 \mu}\left(\beta^{\mu v}-\frac{1}{2} g^{\mu v} \beta\right)=0
$$

and

$$
\begin{gathered}
\beta_{\mu v}=\left[\left(g_{\mu v, 4}\right)_{0}\right] \\
\beta^{\mu v}=g^{\mu \sigma} g^{v \tau} \beta_{\sigma \tau}, \\
\beta=g^{\mu v} \beta_{\mu v} .
\end{gathered}
$$

The result given above, namely, that one may introduce a coordinate system such that the $g_{\mu \nu}$ are continuous across a null-hypersurface and Eqs. (5.11) obtain was derived by Papapetrou and Treder [4] and by Lichnerowicz [5]. The variational derivation given above may be used in any coordinate system. We may also express the conditions derived above in the following geometric terms: The expansion and shear of the vector field $k_{\mu}$ must be continuous across $\Sigma$ as must be the induced "volume" element on $\Sigma$. In addition the vector field $m^{\mu}$ must be such that

and

$$
\begin{aligned}
{\left[m_{; \mu}^{\mu}\right] } & =0 \\
k_{\mu}\left[m^{\mu ; v}\right] & =0 .
\end{aligned}
$$

Eq. (5.12) is equivalent to Eq. (5.6) and Eqs. (5.13) are equivalent to Eqs. (5.7).

\title{
References
}

1. Tabensky, R., Taub, A.H.: Commun. math. Phys. 29, 61-77 (1973).

2. Taub, A.H.: Variational principles in general relativity, Chapter III in Relativistic Fluid Dynamics; Centro Internazionale Mathematico Estivo, Bressanone 7-16 July 1970, Edizioni Cremonese, Roma 1971.

3. Lichnerowicz, A.: Relativistic hydrodynamics and magnetohydrodynamics; New York: W. A. Benjamin, Inc. 1967.

4. Papapetrou, A., Treder,H.: Math. Nachr. 23, 371-384 (1961).

5. Lichnerowicz, A.: Compt. Rend. 273, 528-532 (1971).

\author{
A. H. Taub \\ Mathematics Department \\ University of California \\ Berkeley 4, California 94720 \\ USA
}

